\title{
KINETIC ANALYSIS OF DUCK E-CRYSTALLIN WITH L-LACTATE DEHYDROGENASE ACTIVITY: DETERMINATION OF KINETIC CONSTANTS AND COMPARISON OF SUBSTRATE SPECIFICITY
}

\author{
Chi-Yue Wua ${ }^{a}$, Shui-Tein Chen ${ }^{b}$, Shyh-Horng Chiou ${ }^{a}, b^{*}$ and Kung-Tsung Wang ${ }^{b}, c^{*}$ \\ ${ }^{a}$ Graduate Institute of Biochemical Sciences, National Taiwan University, ${ }^{b}$ Institute of \\ Biological Chemistry, P.O. Box 23-106 Academia Sinica, and ${ }^{c}$ Department of Chemistry, \\ National Taiwan University, Taipei, Taiwan
}

Received June 2, 1992

A systematic analysis of the kinetic properties of duck lens $\varepsilon$-crystallin with lactate dehydrogenase [LDH, (E.C. 1.1.1.27)] activity was carried out by employing some 19 different $\alpha$-keto acids as substrates for this NADH-dependent LDH-catalyzed reaction. The steady-state Michaelis and catalytic constants $\left(\mathrm{K}_{\mathrm{m}}, \mathrm{k}_{\mathrm{cal}}\right)$ were determined for a broad range of organic compounds. The results provide important insights regarding the binding and affinity of substrates to active sites of this enzyme crystallin and indicate a great potential for the application of the stable $\varepsilon$-crystallin as a catalyst to the synthesis of some important chiral $\alpha$-hydroxyacids in a convenient and efficient way. It is also demonstrated for the first time that in addition to the enzymatic activity of lactate dehydrogenase, duck $\varepsilon$-crystallin also possesses the enzymatic activity of malate dehydrogenase.

$\varepsilon$-Crystallin, a relatively abundant lens protein (10-20\% of total lens extract) present in some avian and reptilian species, was shown to possess an L-lactate dehydrogenase activity with high stability suitable for the application to enzyme technology [1]. Lactate dehydrogenase is a "house-keeping" nicotinamide-dependent enzyme in the glycolysis, the major metabolic pathway that metabolizes glucose to lactate and generates the energy in the form of ATP in the absence of oxygen for most organisms [2]. Despite the structural similarities between $\varepsilon$-crystallin and chicken heart-type lactate dehydrogenase, differences in charge and kinetic properties were revealed by isoenzyme electrophoresis and kinetic studies [3]. It was also shown that the L-LDH activity of $\varepsilon$-crystallin were more stable towards thermal denaturation than other commercially available lactate dehydrogenases.

The L-lactate dehydrogenases (L-LDHs) from conventional sources of various animal species have been selected as a model of enzymes with considerable future promise in asymmetric synthesis because of its broad substrate specificity and high enantioselectivity [4,5]. L-LDH was also shown previously to be one of the efficient catalysts that should be useful in the enantioselective reduction of some unnatural 2-oxo acids [6,7]. Since $\varepsilon$ -

\footnotetext{
* To whom correspondence should be addressed.
} 
crystallin can be obtained in high yields very easily and inexpensively from the eye lenses of some avian species, in this report a special effort is further directed to explore the range of some unnatural substrates accepted by $\varepsilon$-crystallin and the stereochemical restraints involved in the binding of substrates to the active site of this enzyme crystallin.

\section{MATERIALS AND METHODS}

\section{General procedure and isolation of e-crystallin}

The isolation and purification procedures for duck $\varepsilon$-crystallin were the same as described previously [8]. Formate dehydrogenase (yeast: E.C. 1.2.1.2.) was obtained in lyophilized form from Boehringer-Mannheim (Mannheim, Germany). Enzymes used for kinetic measurements were dissolved in $30 \mathrm{mM}$ phosphate buffer solution. Pyruvic acid was obtained from E. Merck (Darmstadt, Germany). 3-Fluoropyruvic acid and nercaptopyruvic acid were obtained from Aldrich Chemical Co., Inc. (Milwaukee, WI, USA). Other 2-oxo acids, NADH, and $\mathrm{NAD}^{+}$were obtained from Sigma Chemical Company (St. Louis, MO, USA). Structural confirmation of the products was carried out by the combination of NMR and IR spectroscopies. Chemical shifts of ${ }^{1} \mathrm{H}-\mathrm{NMR}$ and ${ }^{13} \mathrm{C}$ NMR spectra were recorded with a Bruker AM-200 using solvent peaks as references. IR spectra were recorded from $\mathrm{KBr}$ thin films with a Jasco IR-700. UV absorbance was measured in a Hitachi U-2000 spectrophotometer. The optical rotation was measured using a Universal Polarimeter (Schmidt \& Haensch, Berlin, Germany).

Gel electrophoresis of duck $\varepsilon$-crystallin and authentic chicken heart and muscle LDHs

SDS-polyacrylamide slab gel (5\% stacking/ $14 \%$ resolving gel) electrophoresis (SDS-PAGE) was as described with some modifications [9]. The gel was fixed in $12.5 \%$ trichloroacetic acid, washed several times with $20 \%$ methanol/ $7 \%$ acetic acid and stained with Coomassie blue. Native gel ( $8 \%$ resolving gel without stacking) electrophoresis was also adopted for the detection of LDH activity using nitroblue tetrazolium chloride/5methylphenazinium methylsulfate in the coupled oxido-reduction conversion of lactate/NAD and pyruvate/NADH in Tris-glycine buffer of Davis as described before [10].

\section{Kinetic measurements}

The kinetic parameters of $\varepsilon$-crystallin were measured according to the previous report [6]. The L-lactate dehydrogenase activities of $\varepsilon$-crystallin were studied in the direction of reduction in the presence of $\mathrm{NADH}$ in $30 \mathrm{mM}$ phosphate buffer $(\mathrm{pH} 7.2)$ at $25^{\circ} \mathrm{C}$ by measuring the decrease in UV absorbance at $340 \mathrm{~nm}$. The assay solution containing NADH $(0.2 \mathrm{mM})$ and various concentrations of substrates plus different amounts of $\varepsilon$-crystallin was dissolved in $30 \mathrm{mM}$ phosphate buffer ( $\mathrm{pH} \mathrm{7.2)}$ before use. The concentrations of substrates were increased from 0.2 to $5 \mathrm{~K}_{\mathrm{m}}$, and the amount of $\varepsilon$-crystallin was increased by a factor of 100 -fold. The kinetic parameters were obtained from Eadie-Hofstee plots.

\section{RESULTS AND DISCUSSION}

Bioorganic synthesis based on enzymatic catalysis is providing an increasingly important tool in the methodology of enantioselective synthesis of valuable organic intermediates (i.e. chiral synthons) [4-6]. Despite the numerous applications of enzymes for the generation of chiral synthons, the number of enzymes of broad synthetic capability that are readily and inexpensively available is somewhat restricted at present. The LDH group of enzymes is one whose preparation efficacy for chiral synthons has been demonstrated. We have previously demonstrated the facile synthesis of chiral 2-hydroxy 


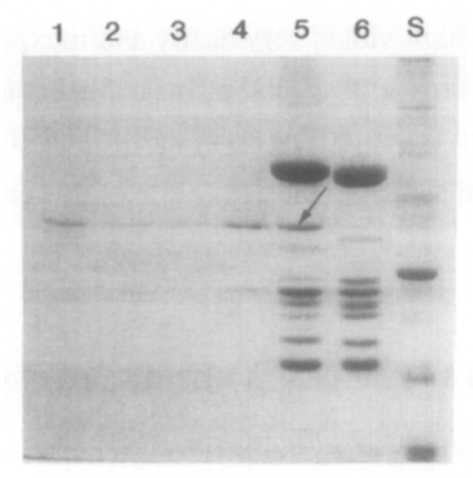

Fig. 1. Gel electrophoresis of duck lens $\mathrm{E}$-crystallin and chicken lactate dehydrogenases (LDHs) under denaturing conditions (SDS-PAGE) in the presence of $5 \mathrm{mM}$ dithiothreitol. Lanes S, standard proteins used as molecular mass markers (in $\mathrm{kDa}$ ): transferrin (80), bovine serum albumin (66), ovalbumin (45), carbonic anhydrase (30), soybean trypsin inhibitor (20) and lysozyme (14). Lane 1, chicken heart LDH; lane 2, chicken muscle LDH; lane 3 , twice purified $\varepsilon$-crystallin; lane $4, \varepsilon$-crystallin fraction from single-step anionexchange chromatography; lane 5, duck lens extract; lane 6, chicken lens extract. The gel was stained with Coomassie Blue. Arrow indicates the electrophoretic position of $\varepsilon$ crystallin with a subunit molecular mass of $37 \mathrm{kDa}$, the major band of $50 \mathrm{kDa}$ above it being $\delta$-crystallin. Note that all native protein samples of lanes $1-5$ showed strong LDH activity-stained bands by native electrophoresis and $\varepsilon$-crystallin is absent in chicken lens.

acids by a naturally abundant and stable duck lens $\varepsilon$-crystallin with endogenous LDH activity [7]. In this report we further explore the range of substrates which can be utilized by this unique enzymatic crystallin.

\section{Isolation and comparison of duck $\varepsilon$-crystallins and authentic chicken LDHs}

By adopting the anion-exchange chromatography $\varepsilon$-crystallin with a purity of about 90 $\%$ could be easily separated from other classes of crystallins in a single-step separation and purification [3,8]. The identification of $\varepsilon$-crystallin as an LDH-like protein was previously demonstrated by native isoenzyme electrophoresis based on activity staining for LDH $[8,10]$ and SDS-polyacrylamide gel electrophoresis as shown in Fig. 1. Rechromatography of the $\varepsilon$-crystallin fraction on the same column usually obtained over $99 \%$ pure $\varepsilon$-crystallin (lane 3 of Fig. 1). $\varepsilon$-Crystallin showed a protein band of $37.5 \mathrm{kDa}$ in contrast to a major $\delta$ crystallin with a $50 \mathrm{kDa}$ subunit. We have also compared the subunit structure of $\varepsilon$ crystallin with those heart and muscle LDHs from chicken (lancs 1 and 2 of Fig. 1). They were almost indistinguishable regarding the molecular sizes of native and subunit structures [10]. It is to be noted that in contrast to that found in the duck and caiman lenses $\varepsilon$ crystallin cannot be detected in the chicken lens [11,12]. The distinct difference in the expression of $\varepsilon$-crystallin in some species of Anatidae family and not in other poultry birds has attracted a lot of attention recently [13]. The practical use of this stable crystallin plus $\delta$ crystallin to chiral synthesis has been our research focus during the past few years $[7,14]$.

\section{Kinetic analysis and substrate specificity}

To explore the range of substrates accepted by $\varepsilon$-crystallin, various 2-oxo acids with different side-chains were used in the $\mathrm{LDH}$ reaction catalyzed by $\varepsilon$-crystallin in $30 \mathrm{mM}$ phosphate buffer at $\mathrm{pH} 7.2$ and $25^{\circ} \mathrm{C}$ in the direction of pyruvate reduction (Table 1). Fig. 
Table 1. Kinetic parameters for $\mathrm{E}$ crystallin and L-lactate dehydrogenases from different sources $\left(\mathrm{pH} 7.2,25^{\circ} \mathrm{C}\right.$ )

(Values relative to that for reduction of pyruvate to L-lactate) ${ }^{\mathrm{a}}$

\begin{tabular}{|c|c|c|c|c|c|c|c|c|c|c|c|c|c|c|c|c|c|c|c|}
\hline \multirow{2}{*}{\multicolumn{2}{|c|}{ compd }} & \multicolumn{3}{|c|}{$\varepsilon$-crystallin } & \multicolumn{3}{|c|}{ rabbit muscle ${ }^{b}$} & \multicolumn{3}{|c|}{ porcine heart } & \multicolumn{3}{|c|}{ bovine heart' } & \multicolumn{3}{|c|}{ chicken liver $^{b}$} & \multicolumn{3}{|c|}{ lobster tail $^{b}$} \\
\hline & & $\mathrm{Km}$ & Kcat & $\frac{\mathrm{Kcat}}{\mathrm{Km}}$ & $\mathrm{Km}$ & Keat & $\frac{\mathrm{K} \text { cat }}{\mathrm{Km}}$ & $\mathrm{Km}$ & Kcat & $\frac{K_{c a t}}{K_{m}}$ & $\mathrm{Km}$ & Keat & $\frac{\mathrm{Kcat}}{\mathrm{Km}}$ & $\mathrm{Km}$ & Kcat & $\frac{\text { Kcat }}{\mathrm{Km}}$ & $\mathrm{Km}$ & Kcat & $\frac{\mathrm{Kcat}}{\mathrm{Km}}$ \\
\hline 1 & $\mathbf{H}$ & 25 & 360 & 4.5 & 18 & 60 & 1 & 3.2 & 60 & 2 & & 80 & & 3.5 & 110 & 2 & 14 & 70 & 0.7 \\
\hline 2 & $\mathrm{CH}_{3}$ & 0.32 & 100 & 100 & 0.25 & 100 & 100 & 0.09 & 100 & 100 & 0.05 & 100 & 100 & 0.05 & 100 & 100 & 0.13 & 100 & 100 \\
\hline 3 & $\mathrm{CH}_{3} \mathrm{CH}_{2}$ & 4.1 & 200 & 16 & 7.4 & 50 & 2 & 0.6 & 50 & 8 & 0.08 & 60 & 40 & 0.5 & 55 & 6 & 2.2 & s5 & 3 \\
\hline 4 & $\mathrm{CH}_{3} \mathrm{CH}_{2} \mathrm{CH}_{2}$ & 9.1 & 15 & 0.53 & 53 & 1 & 0.05 & 1.9 & 6 & 0.3 & 33 & 6 & 0.09 & 1.0 & 4 & 0.2 & 5.2 & $y$ & 0.2 \\
\hline 5 & $\mathrm{CH}_{3}\left(\mathrm{CH}_{2}\right)_{2} \mathrm{CH}_{2}$ & 15 & 20 & 0.44 & 3.7 & 1 & 0.07 & 1.9 & 7 & 0.3 & 3.0 & 2 & 0.03 & 1.7 & 7 & 0.2 & 4.1 & 10 & 0.3 \\
\hline 6 & $\left(\mathrm{CH}_{3}\right)_{2} \mathrm{CH}$ & 29 & 0.78 & 0.009 & & 0.04 & & 3.9 & 0.3 & 0.007 & & 0.4 & & & 0.4 & & & 0.3 & \\
\hline 7 & $\left(\mathrm{CH}_{3}\right)_{2} \mathrm{CHCH}_{2}$ & 6.2 & 0.019 & 0.001 & & $<0.01$ & & 3.6 & 0.02 & 0.0005 & & 0.01 & & & 0.03 & & & & \\
\hline 8 & $\mathrm{CH}_{3} \mathrm{CH}_{2} \mathrm{CH}\left(\mathrm{CH}_{3}\right)$ & 13 & 0.034 & 0.0009 & & $<0.01$ & & 5.2 & 0.01 & 0.0002 & & 0.01 & & & 0.01 & & & & \\
\hline 9 & $\mathrm{FCH}_{2}$ & 0.46 & 59 & 41 & 0.89 & 80 & 22 & 0.08 & 40 & 45 & 0.1 & 30 & 15 & 0.6 & 50 & 4 & 0.2 & 40 & 30 \\
\hline 10 & $\mathrm{HOCH}_{2}$ & 1.3 & 200 & 48 & 1.0 & 120 & 30 & 0.2 & 170 & 80 & & 100 & & 0.2 & 160 & 30 & 1.7 & 110 & 8 \\
\hline 11 & $\mathrm{HSCH}_{2}$ & 3.0 & 66 & 7.1 & 0.6 & 20 & 8 & 0.4 & 60 & 15 & & 100 & & 0.3 & 70 & 6 & 0.4 & 20 & 7 \\
\hline 12 & $\mathrm{CH}_{3} \mathrm{COCH}_{2}$ & 1.6 & 4.4 & 0.9 & 3.9 & 2 & 0.1 & 0.8 & 3 & 0.3 & 0.4 & 10 & 1 & 0.8 & 3 & 0.2 & 0.4 & 2 & 0.7 \\
\hline 13 & $\mathrm{CH}_{3} \mathrm{SCH}_{2} \mathrm{CH}_{2}$ & 17 & 7.4 & 0.14 & 5.4 & 0.3 & 0.01 & 3.4 & 2 & 0.05 & & 6 & & 4.9 & 2 & 0.03 & 5.7 & 3 & 0.07 \\
\hline 14 & $\mathrm{C}_{6} \mathrm{H}_{5} \mathrm{CH}_{2}$ & 6.6 & 6.4 & 0.31 & 6.5 & 0.6 & 0.02 & 2.2 & 2 & 0.08 & & 3 & & 1.7 & 2 & 0.06 & 4.1 & 8 & 0.3 \\
\hline 15 & HOOC & 35 & 20 & 0.18 & & & & & & & & & & & & & & & \\
\hline 16 & $\mathrm{HOOCCH}_{2}$ & 8.2 & 103 & 4.0 & & & & & & & & & & & & & & & \\
\hline 17 & $\mathrm{HOOCCH}_{2} \mathrm{CH}_{2}$ & 140 & 0.27 & 0.0006 & & & & & & & & & & & & & & & \\
\hline 18 & $\mathrm{HOOCCH}_{2} \mathrm{CH}_{2} \mathrm{CH}_{2}$ & 38 & 0.26 & 0.002 & & & & & & & & & & & & & & & \\
\hline 19 & Imidazolyl $-\mathrm{CH}_{2}$ & 22 & 1.2 & 0.017 & & & & & & & & & & & & & & & \\
\hline
\end{tabular}

"The specific activities for reduction of pyruvate (units per mg of enzyme) are $350,160,250,270,365,190 \mu \mathrm{mol} \mathrm{min}^{-1} \mathrm{mg}^{-1}$, respectively, for $\varepsilon$-crystallin, rabbit muscle, porcine heart, bovine heart, chicken liver, and lobster tail L-lactate dehydrogenases.

${ }^{\mathrm{b}} \mathrm{Km}$ is expressed in $\mathrm{mM}, \mathrm{Kcat}$ and $\mathrm{Kcat} / \mathrm{Km}$ are relative ratios based on pyruvate as 100 (cited in [6]).

2 shows representative kinetic data corresponding to Eadie-Hofstee plots for the reactions of 2-hydroxypyruvate and 2-fluoropyruvate, the correlation coefficient being $>0.99$ in each case. The reported values of kinetic data from other sources of lactate dehydrogenases were cited from the studies of others [6]. Although the values of $\mathrm{Km}$ of duck $\varepsilon$-crystallin are slightly larger than those of other LDHs of different sources, the values of Kcat of $\varepsilon$ crystallin are significantly larger than those catalytic constants reported for LDHs of other sources for various substrates.

The most interesting observation in Table 1 is that the values of Kcat of compounds 1 , 3 , and 10 are larger than that for the natural substrate of pyruvate, as summarized in Table 2. Moreover, $\varepsilon$-crystallin possessed high enzyme activity for the reduction of oxaloacetic acid (Table 1, compound 16). This result indicated that it had not only L-lactate dehydrogenase activity but also malate dehydrogenase activity. In order to investigate the specificity of this latter enzymatic activity, we have selected three 3-oxo acids and one 4oxo acid (Table 2) as the substrates for $\varepsilon$-crystallin. Results indicated that $\varepsilon$-crystallin possessed no catalytic activity in the oxido/reduction of 3-oxo and 4-oxo acids, which corroborated that the high specificity of $\varepsilon$-crystallin is to convert oxaloacetate to malate. The biological significance of this finding remains an interesting topic for future investigation.

From the consideration of different molecular sizes of side chains and the relative affinities, as reflected in different $\mathrm{Km}$ for 19 compounds (Tables 1 and 2), it can be deduced that $\varepsilon$-crystallin possesses some stereochemical restraints regarding the substrate 


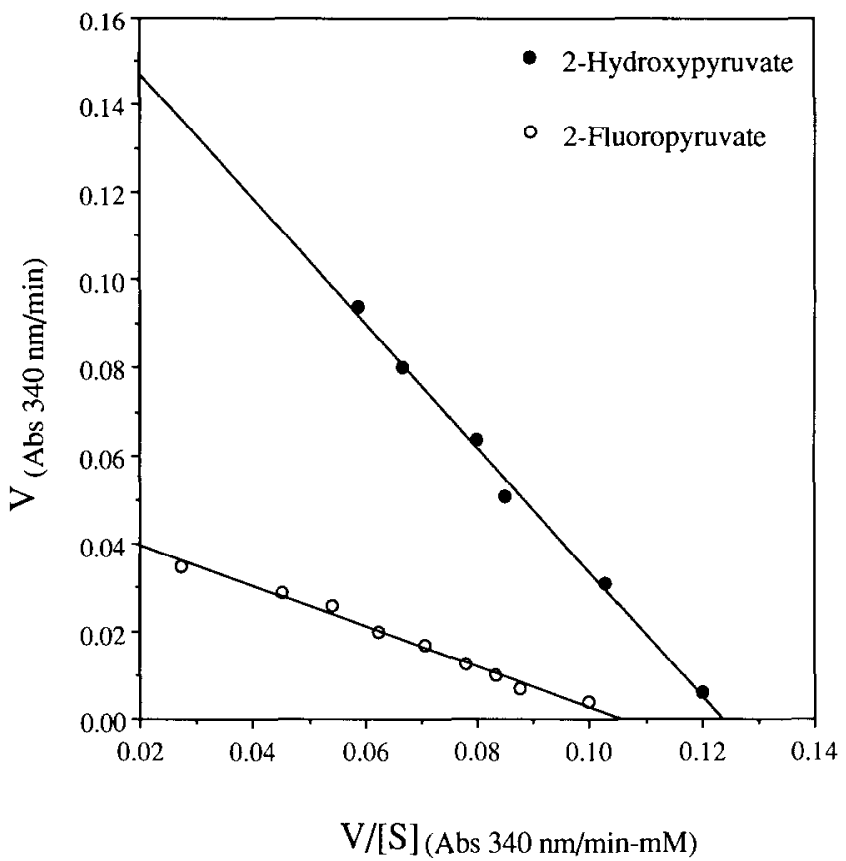

Fig. 2. Determination of kinetic parameters by Eadie-Hofstee plots for the reduction of two 2-oxo acids (compounds 9 and 10 in Table 1 ) catalyzed by purified duck $\varepsilon$-crystallin. The L-lactate dehydrogenase activities of $\varepsilon$-crystallin were studied in the direction of reduction in the presence of NADH in $30 \mathrm{mM}$ phosphate buffer $(\mathrm{pH} 7.2)$ at $25^{\circ} \mathrm{C}$ by measuring the decrease in UV absorbance at $340 \mathrm{~nm}$. The assay solution containing NADH $(0.2 \mathrm{mM})$ and various concentrations of substrates was dissolved in $30 \mathrm{mM}$ phosphate buffer ( $\mathrm{pH} 7.2$ ) before use. The concentrations of substrates were increased from 0.2 to 5 $\mathrm{K}_{\mathrm{m}}$, and the amount of $\varepsilon$-crystallin was increased by a factor of 100 -fold. The $\mathrm{Km}$ 's and Kcat's determined from the plots are 0.46 and $1.3 \mathrm{mM}$ and 96.2 and $326 \mathrm{~min}^{-1}$ for 2fluoropyruvate (compound 9) and 2-hydroxypyruvate (compound 10), respectively.

binding and affinity to the enzymatic active site. By comparing the $\mathrm{Km}$ 's for the pairs of compounds 3 and 6 plus 4 and 8, the side chain of A carbon as depicted in Fig. 3 is expected to be a small nonpolar group to get a high-affinity binding to the enzyme. By analogy, from the determined Km's of compounds 4 and 12, large polar side chain of B carbon is favorable for the enzyme binding. The comparison of $\mathrm{Km}$ 's for compounds 4 and 17 plus 8 and 18 led to the deduction that side chains of $C$ and $D$ carbons of the substrates favor small and large nonpolar groups respectively. In summary the side chains of $\alpha$-keto acids with good binding can contain one large hydrophilic or charge group at $\boldsymbol{\gamma}$-carbon which may account for the binding of oxaloacetate to $\varepsilon$-crystallin. However the $\beta$-, $\delta$ - and $\varepsilon$-carbon containing side-chains are in general more favorable for hydrophobic groups.

\section{Application of $\varepsilon$-crystallin to synthesis of $(S)$-2-hydroxy acids}

Because $\varepsilon$-crystallin can adopt a broad range of substrates and maintain its activity in pure water and room temperature for a long period of time [7]. We have selected 2oxopentanoic acid, one of the precursors for the synthesis of pharmaccutically important intermediates ( $\mathbf{S}$ )-2-hydroxy acids, as substrate to demonstrate $\varepsilon$-crystallin as a catalyst for a practical-scale synthesis of chiral compounds. The product, (S)-2-hydroxypentanoic acid, 
Table 2. Comparison of relative reactivities of different oxo acids in $\varepsilon$-crystallin catalyzed reductions

(A) 2-Oxo Acids<smiles>[O]C(=O)C(=O)O</smiles>

(1)

R group : $\quad$ Reactivity Kcat $^{\text {a }}$

(2)

$\begin{aligned} \mathrm{H} & \\ \mathrm{HOCH}_{2} & \text { Very Good } \\ \mathrm{CH}_{3} \mathrm{CH}_{2} & >100 \% \\ \mathrm{HOOCCH} & \end{aligned}$

)

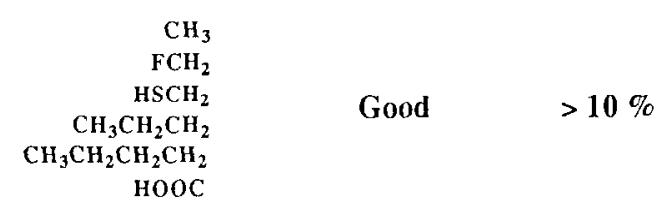

(3)

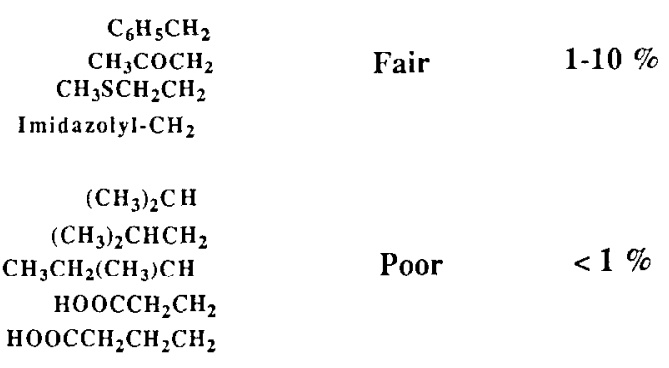

(B) 3-Oxo and 4-Oxo Acids

$\begin{array}{r}\mathrm{CH}_{3} \mathrm{COCH}_{2} \mathrm{COOH} \\ \mathrm{CH}_{3} \mathrm{COCH}_{2} \mathrm{CH}_{2} \mathrm{COOH} \\ \mathrm{HOOCCH}_{2} \mathrm{COCH}_{2} \mathrm{COOH} \\ \mathrm{HOOCCH} \mathrm{CH}_{2} \mathrm{COCH}_{2} \mathrm{COOH}\end{array}-$ No Reaction
-

${ }^{a}$ Kcat values for different substrates are relative ratios based on that of pyruvate as 1.

a chiral intermediate in the synthesis of various fungicides, has been synthesized in high yields and optical purity utilizing an in situ NADH regeneration system of duck $\varepsilon$-crystallin coupled with formate/formate dehydrogenase. The product was identified as pure compound based on spectroscopic data of ${ }^{1} \mathrm{H}$ NMR, ${ }^{13} \mathrm{C}$ NMR and IR spectra, with no further purification required [ 7 and unpublished results].

In conclusion we have explored the substrate specificity and kinetic constants of various $\varepsilon$-crystallin catalyzed LDH-type oxido/reduction reactions. It is demonstrated that this natural LDH mutant present in the eye lenses of some avian and reptilian species possesses a broader range of substrates than other lactate dehydrogenases of conventional sources. Therefore, it offers significant advantages in the application of this enzyme crystallin as a bioorganic catalyst in the synthesis of various 2-hydroxy acids. It is also demonstrated for the first time that in addition to the enzymatic activity of lactate dehydrogenase, duck $\varepsilon$-crystallin also possesses the enzymatic activity of malate 


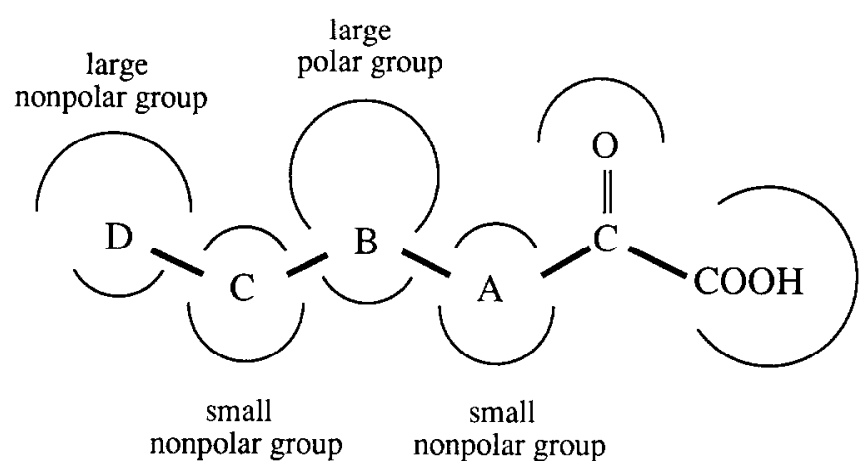

Fig. 3. The substrate binding site of $\varepsilon$-crystallin in relation to the stereochemical properties of side-chain groups on various $\alpha$-keto acids compounds. Note that the deduced large polar binding site for carbon-B side chain ( $\gamma$-carbon position for $\alpha$-keto acids) may explain the possession of malate dehydrogenase activity in duck lens $\varepsilon$-crystallin.

dehydrogenase. The biological significance of this dual-function crystallin may shed some light on the evolution of lens crystallins and its possible metabolic functions in general.

\section{ACKNOWLEDGMENTS}

This work was supported in part by Academia Sinica and the National Science Council, Taipei, Taiwan. Thanks are also due for the kind provision of duck lenses by the Taiwan Livestock Research Institute.

\section{REFERENCES}

[1] Chiou, S.-H. , Lee, II.-J. \& Chang, G.-G. (1990) Biochem. J. 267, 51-58.

[2] Metzler, D.E. (1977) In Biochemistry: The chemical reactions of living cells. Academic Press, New York.

[3] Chiou, S.-H., Chang, W.-P. \& Chen, C.-C. (1989) Biochem. International 18, 1093-1100.

[4] Whitesides, G.M. \& Wong, C.-H. (1985) Angew. Chem., Int. Ed. Engl. 24, 617 638.

[5] Luyten, M. A., Bur, D., Wynn, H., Parris, W., Gold, M., Friesen, J. D. \& Jones, J. B. (1989) J. Am. Chem. Soc. 111, 6800-6804.

[6] Kim, M.-J. \& Whitesides, G.M. (1988) J. Am. Chem. Soc. 110, 2959-2964.

[7] Wu, C.-Y., Chen, S.-T., Chiou, S.-H. \& Wang, K.-T. (1992) FEBS Lett. 301, 219-222.

[8] Chiou, S.-H. \& Chang, W.-P. (1989) In International Symposium on Biologically Active Proteins and Peptides (Eds., S.-H. Chiou, K.-T. Wang \& S.-H. Wu), pp. 81-89, Academia Sinica, Taipei, Taiwan.

[9] Laemmli, U.K. (1970) Nature 227, 680-685.

[10] Chiou, S.-H., Chang, W.-P. \& Lin, H.-K. (1988) Biochim. Biophys. Acta 957, 313-317.

[11] Chiou, S.-H., Lee, H.-J. \& Chang, G.-G. (1990) Biochem. J. 267, 51-58.

[12] Chiou, S.-H., Lee, H.-J., Huang, S.-M. \& Chang, G.-G. (1991) J. Protein Chem. 10, 161-166.

[13] Wistow, G.J., Mulders, J.W.M. \& de Jong, W.W. (1987) Nature 326, 622-624.

[14] Wu, C.-Y., Chen, S.-T., Chiou, S.-H. \& Wang, K.-T. (1991) Biotech. Lett. 13, 405410 . 